\title{
News and views into the SNARE complexity in Arabidopsis
}

\author{
Sang-Jin Kim ${ }^{1,2}$ and Federica Brandizzi 1,2,3* \\ ' Great Lakes Bioenergy Research Center, Michigan State University, East Lansing, MI, USA \\ 2 Plant Research Laboratory, Department of Energy, Michigan State University, East Lansing, MI, USA \\ ${ }^{3}$ Department of Plant Biology, Michigan State University, East Lansing, MI, USA
}

Edited by:

Robert Mullen, University of Guelph,

Canada

Reviewed by:

Jurgen Denecke, University of Leeds, UK

Mary Lai Preuss, Webster University, USA

\section{${ }^{*}$ Correspondence:}

Federica Brandizzi, Plant Research

Laboratory, Department of Energy,

Michigan State University, East

Lansing, MI 48824, USA.

e-mail: fb@msu.edu

\begin{abstract}
Secretory organelles are engaged in a continuous flux of membranes, which is believed to occur mostly via transport vesicles. Being critical in maintaining several cellular functions, transport vesicles are membrane-enclosed sacs that temporarily store and then deliver membrane lipids, protein, and polysaccharides. SNAREs have a crucial role in vesicle traffic by driving membrane fusion and conferring fidelity through the formation of specific SNARE complexes. Additionally, specific roles of SNAREs in growth and development implicate that they are versatile components for the life of a plant. Here, we summarize the recent progress on the understanding of the role of SNAREs and highlight some of the questions that are still unsolved.
\end{abstract}

Keywords: Arabidopsis, SNARE, vesicle trafficking, membrane fusion

\section{INTRODUCTION}

Generally, vesicle trafficking occurs via three important steps: vesicle budding from the donor organelle, movement towards a target organelle, and fusion. Several proteins are involved in docking and fusion of vesicles for target recognition and driving membrane fusion (Malsam et al., 2008). Among the proteins for vesicle trafficking, SNAREs (soluble $N$-ethylmaleimide-sensitive fusion protein attachment protein receptors) were described a critical role in membrane fusion (Sollner et al., 1993). SNAREs can be categorized into two types: v-SNAREs, as SNAREs on the vesicle, and t-SNAREs, as SNAREs on the target membrane. When a vesicle moves close to its target, a v-SNARE on the vesicle interacts with three t-SNAREs on the target membrane, forming a hetero-tetrameric trans-SNARE complex that drives membrane fusion (McNew et al., 2000). According to the core amino acid in the hydrophobic heptad repeats in the SNARE motif, SNAREs can be divided into four groups: Qa-, Qb-, Qc-, and R-SNAREs (Fasshauer et al., 1998). In mammals, a typical SNARE complex consists of Qa- (syntaxin 1 like), Qb- (N-terminal half of SNAP25 like), and Qc- (C-terminal half of SNAP25 like) SNAREs on the target membrane, and R-SNAREs on the vesicle (Bock et al., 2001). The same combination seems to be conserved also in plants (Sutter et al., 2006a). It has been reported that there may be as many as 65 SNAREs in Arabidopsis (Table 1; Sanderfoot, 2007; Saito and Ueda, 2009). A large number of SNAREs in Arabidopsis may help facilitate trafficking in a complex endomembrane system that include distinct secretory and vacuolar trafficking steps mediated by two times more SNAREs than unicellular or mammalian systems (Sanderfoot, 2007). Studies to date on the same family of plant SNAREs have often revealed redundancy but also functional specificity. Here, we summarize the roles of SNAREs in Arabidopsis and present additional thoughts for future SNARE studies.

\section{ROLE OF ER AND GOLGI SNARES}

In the endoplasmic reticulum (ER) and Golgi apparatus, 21 SNAREs (three Qa-SNAREs: AtSYP81 and two AtSYP3; seven Qb-SNAREs: AtSEC20, two AtMEMB1, two AtGOS1, and two AtUSE1; seven Qc-SNAREs: three AtSYP7, two AtBS14, and two AtSFT1; four R-SNAREs: two AtSEC22, AtVAMP714, and AtVAMP723) have been localized at a subcellular level by microscopy with fluorescent protein fusions along with in silico analyses (Uemura et al., 2004; Chatre et al., 2005; Sanderfoot, 2007; Bubeck et al., 2008). In general, a Qa-SNARE is the core SNARE that regulates a SNARE complex formation at the target membrane. Interestingly, several Qa-SNARE null mutants have been reported to be lethal, supporting that Qa-SNAREs are essential (Sanderfoot et al., 2001b). Two Qa-SNAREs (AtSYP81 and AtSYP31) have been shown to be involved in ER-Golgi traffic (Bubeck et al., 2008). When secretory and ER retention markers were used to investigate alteration of ER-Golgi traffic, it was found that AtSYP81 overexpression inhibited both anterograde and retrograde membrane traffic, while AtSYP31 overexpression only inhibited anterograde traffic (Bubeck et al., 2008). In yeast, the ortholog of AtSYP31 has been reported to interact with the yeast counterparts of AtSEC22 and AtMEMB11 (Tsui et al., 2001). Interestingly, in tobacco leaf epidermis a strong redistribution of Golgi proteins into the ER was also observed in conditions of overexpression of AtSEC22 or AtMEMB11 (Chatre et al., 2005), implicating also these proteins in traffic at the ER/Golgi interface. At least for AtSEC22, this may be a conserved function. For example, yeast Sec22p was found to have a role in both anterograde and retrograde traffic between ER and Golgi (Spang and Schekman, 1998). Furthermore, components of the COPII machinery in mammalian cells, Sec24a and Sec24b, were found to interact directly with Sec22p (Mancias and Goldberg, 2008). In plants, we do not know whether AtSEC22 interacts with either COPI or 
Table 1 | Localization of SNAREs.

\begin{tabular}{|c|c|c|c|}
\hline & ER/Golgi & $\begin{array}{l}\text { Dual } \\
\text { localization }\end{array}$ & Reference \\
\hline \multirow[t]{3}{*}{$\mathrm{Qa}$} & AtSYP81 & & \\
\hline & AtSYP31 & & \\
\hline & AtSYP32 & & \\
\hline \multirow[t]{7}{*}{$\mathrm{Qb}$} & AtSEC20 & & \\
\hline & AtMEMB11 & & \\
\hline & AtMEMB12 & & \\
\hline & AtGOS11 & & \\
\hline & AtGOS12 & & \\
\hline & AtUSE11 & & \\
\hline & AtUSE12 & & \\
\hline \multirow[t]{7}{*}{ Qc } & AtSYP71 & PM/endosome & Suwastika et al. (2008) \\
\hline & AtSYP72 & & \\
\hline & AtSYP73 & & \\
\hline & AtBS14a & & \\
\hline & AtBS14b & & \\
\hline & AtSFT11 & & \\
\hline & AtSFT12 & & \\
\hline \multirow[t]{4}{*}{$\mathrm{R}$} & AtSEC22 & & \\
\hline & AtSEC22-like & & \\
\hline & AtVAMP714 & & \\
\hline & AtVAMP723 & & \\
\hline \multicolumn{4}{|c|}{ TGN/ENDOSOME/VACUOLE } \\
\hline \multirow[t]{6}{*}{ Qa } & AtSYP41 & & \\
\hline & AtSYP42 & & \\
\hline & AtSYP43 & & \\
\hline & AtSYP21 & PVC/vacuole & Uemura et al. (2010) \\
\hline & AtSYP22 & PVC/vacuole & Uemura et al. (2010) \\
\hline & AtSYP23 & $\begin{array}{l}\text { Cytosol/PVC/ } \\
\text { vacuole }\end{array}$ & Shirakawa et al. (2010) \\
\hline \multirow[t]{4}{*}{$\mathrm{Qb}$} & AtVTI11 & PVC/vacuole & Ebine et al. (2008) \\
\hline & AtVTI12 & & \\
\hline & AtVTI13 & & \\
\hline & AtVTI14 & & \\
\hline \multirow[t]{5}{*}{ Qc } & AtSYP61 & $\begin{array}{l}\text { TGN/endosome/ } \\
\text { PM }\end{array}$ & Drakakaki et al. (2011) \\
\hline & AtSYP51 & PVC/vacuole & $\begin{array}{l}\text { Sanderfoot et al. (2001a), } \\
\text { Ebine et al. (2008) }\end{array}$ \\
\hline & AtSYP52 & PVC/vacuole & Sanderfoot et al. (2001a) \\
\hline & AtSYP5-like & & \\
\hline & AtSYP5-like & & \\
\hline \multirow[t]{6}{*}{$\mathrm{R}$} & AtYKT61 & & \\
\hline & AtYKT62 & & \\
\hline & AtVAMP711 & & \\
\hline & AtVAMP712 & & \\
\hline & AtVAMP713 & & \\
\hline & AtVAMP727 & $\begin{array}{l}\text { Endosome/ } \\
\text { PM/vacuole }\end{array}$ & Ebine et al. (2011) \\
\hline \multicolumn{4}{|c|}{ PLASMA MEMBRANE } \\
\hline \multirow[t]{3}{*}{ Qa } & AtSYP111 & & \\
\hline & AtSYP112 & & \\
\hline & AtSYP121 & & \\
\hline
\end{tabular}

(Continued)
Table 1 | Continued

\begin{tabular}{lll}
\hline ER/Golgi & $\begin{array}{l}\text { Dual } \\
\text { localization }\end{array}$ & Reference \\
\hline & AtSYP122 \\
& AtSYP123 \\
& AtSYP124 \\
& AtSYP125 \\
& AtSYP131 \\
& AtSYP132 \\
Ob & AtNPSN11 \\
& AtNPSN12 \\
& AtNPSN13 \\
Ob+ Qc AtSNAP29 \\
AtSNAP30 \\
AtSNAP33 \\
AtVAMP721 \\
AtVAMP722 \\
AtVAMP723 \\
AtVAMP724 \\
AtVAMP725 \\
AtVAMP726 \\
TOMOSYNa \\
TOMOSYNb
\end{tabular}

Localization of SNARES are either experimentally investigated or predicted (Uemura et al., 2004; Sanderfoot, 2007; Saito and Ueda, 2009). SNAREs with multiple localization are specified in the table with references.

COPII; however, an essential role in ER/Golgi membrane traffic has been further supported by analyses of loss-of-function mutants. Specifically, the atsec22 mutation was reported to affect Golgi morphology and cause ER retention of a plasma membrane (PM) SNARE in pollen (El-Kasmi et al., 2011). Similarly to mammalian cells, AtSEC22 might interact with COPII coat components; however, in general, an interaction between coat proteins and SNAREs has not been reported in plants yet. The Arabidopsis genome encodes a large number of genes for coat proteins, often outnumbering other eukaryotes isoforms (Robinson et al., 2007). Possibly, specific SNARE-COPII protein interactions may at least partially explain the diversification of the ER and Golgi SNAREs and coat proteins in plants.

Evidence is emerging that some of the plant SNAREs may have dual localization. For example, AtSYP71 is distributed both at the ER and PM. Although in conditions of overexpression AtSYP71 was found in the ER, AtSYP71 expressed using its endogenous promoter was found to localize at PM in the Arabidopsis root as well as ER and PM localization in the dividing root cells (Suwastika et al., 2008). This suggests that SYP71 might form at least two different SNARE complexes on the ER and PM, or that AtSYP71 is a SNARE for the ER-PM traffic during cell division. No AtSYP71 homolog has been identified in mammals, suggesting that AtSYP71 may be involved in plant-specific membrane traffic events. Thus, identification of other SNAREs in the AtSYP71 SNARE complex may clarify the nature of the plant-specific role of AtSYP71, perhaps by providing important insights on understudied traffic route(s) that may occur between the ER and PM. 
Two types of electron-dense vesicles originating from the ER have been suggested for the transport of storage proteins or proteases to storage or lytic vacuoles in pumpkin seeds and castor bean (Hara-Nishimura et al., 1998; Toyooka et al., 2000; Hayashi et al., 2001). However, trafficking components required for budding and cargo selection in such traffic routes have not been identified yet. Proteomics analyses of these dense vesicles may reveal important clues on the machinery and SNAREs that allows membrane transport to plant-specific organelles such as the protein storage vacuole.

\section{ROLE OF TGN, ENDOSOMAL, AND VACUOLAR SNARES}

At the TGN, endosome, and vacuoles, 21 SNAREs (six Qa-SNAREs: three AtSYP4 and three AtSYP2; four Qb-SNAREs: AtVTI1; five Qc-SNAREs: AtSYP61, three AtSYP5, and two AtSYP5-like proteins; six R-SNAREs: two AtYKT6, three AtVAMP71, and AtVAMP727) have been identified (Uemura et al., 2004; Saito and Ueda, 2009; Shirakawa et al., 2010). The TGN is a tubular and vesicular organelle facing the trans side of the Golgi apparatus. Mounting evidence supports that the TGN represents a crossroad for post-Golgi protein traffic because it appears that vesicles containing vacuolar or secretory cargo can bud from the TGN, but also that endocytic markers such as the dye FM4-64 can be internalized to the TGN (Nakamura et al., 1993; Ahmed et al., 1997; Dettmer et al., 2006; Toyooka et al., 2007; Scheuring et al., 2011). Thus, complex vesicle budding and fusion events take place at the TGN, and it is likely that membrane transport is highly regulated at this organelle. In this view, specific SNAREs should facilitate anterograde or retrograde traffic between the TGN and endosomes, vacuoles, and PM. In particular, two Qa-SNAREs, AtSYP41 and AtSYP42 were found to form separate complexes in distinct TGN domains (Bassham et al., 2000). Furthermore, the corresponding knock-out mutants were found to be gametophytic lethal, suggesting that these SNAREs have specific and essential functions (Sanderfoot et al., 2001b). Recently, another SNARE, AtSYP61, has been implicated in traffic at the TGN. AtSYP61 is a component of the AtSYP4 SNARE family complexes and it is involved in salt and drought stress tolerance (Sanderfoot et al., 2001a; Zhu et al., 2002). Proteomic analysis of vesicles containing AtSYP61 has identified trafficking components at the TGN and PM along with enzyme cargos (Drakakaki et al., 2011). These results suggest that AtSYP61 has possibly a dual role as a t-SNARE at the TGN but also as a SNARE for TGN-PM traffic. Previously, AtSYP42 was co-immunoprecipitated with AtSYP61 (Sanderfoot et al., 2001a), but AtSYP42 was not found in the proteomic analysis in contrast to previous results. It would be interesting however to assay the proteome of the vesicles containing either AtSYP41 or AtSYP42 because we may learn about the function of AtSYP41 and AtSYP42, and, perhaps, determine whether the TGN exists as a uniform compartment or whether there may be TGNs with different composition due to specific functions or maturation stages.

Other SNAREs have been localized in post-Golgi routes. For example, three AtSYP2 family members, AtSYP21, AtSYP22, and AtSYP23, have been shown to localize on the prevacuolar compartment (PVC), vacuole, and cytoplasm, respectively. Individual single mutants are viable, with only a gravitropic defect reported for the atsyp22 mutant (Yano et al., 2003). However, the atsyp21/atsyp22 double mutant is known to be lethal (Shirakawa et al., 2010). A complete complementation of atsyp22 with AtSYP21 under AtSYP22 promoter suggests that AtSYP21 and AtSYP22 may have redundant functions (Uemura et al., 2010). Overexpression of SYP21 resulted in homotypic fusion of PVC as well as the accumulation of vacuolar cargo in the PVC and partial secretion of vacuolar proteins (Foresti et al., 2006), suggesting that overexpression of SYP21 inhibits anterograde trafficking in the PVC-vacuole route. These observations open the question on the molecular basis for the role of AtSYP21 in two different biological events. In other words, it would be interesting to test whether vacuolar trafficking defects and homotypic fusion of PVC observed in conditions of AtSYP21 overexpression are due to the collapse of one or multiple fusogenic machineries. AtSYP23 has a similar protein sequence with AtSYP21, but it does not have transmembrane domain. AtSYP23 can make a SNARE complex with AtVTI11 and AtSYP5, and high expression of AtSYP23 can complement an atsyp22 mutant (Shirakawa et al., 2010). Thus, AtSYP23 may function in vacuolar trafficking like AtSYP21 and AtSYP22. Considering the lack of transmembrane domain in AtSYP23, AtSYP23 may function by specific manner differently from other QaSNAREs. Therefore, either other regulatory proteins may recruit AtSYP23 at the active site for the SNARE complex formation or that AtSYP23 may interact with a preformed SNARE complex. In vitro liposome fusion assays may allow distinguishing the two models.

Similar to AtSYP61, members of the AtVAMP71 family (AtVAMP711, AtVAMP712, AtVAMP713, and AtVAMP714) have been found to be involved in drought stress response by regulating stoma closure (Leshem et al., 2010). Stoma aperture is directly regulated by the volume of the vacuole (Blatt, 2000; Sirichandra et al., 2009). Therefore, the AtVAMP71 family may be involved in membrane fusion to tonoplast or vesicle budding from vacuole so as to control the size of vacuole through modulating the tonoplast size. So far, the composition of the AtVAMP71 family complexes has not been determined, but the presence of three AtVAMP71 SNAREs (AtVAMP711, AtVAMP712, AtVAMP713) on the tonoplast suggests that these SNAREs could have either overlapping functions or that they may form distinct SNARE complexes on the tonoplast for tissue- and developmental-specific roles. Another RSNARE, AtVAMP727 is localized on the endosome/PVC and PM (Uemura et al., 2004; Ebine et al., 2008, 2011). It forms a complex with AtSYP22, AtVTI11, and AtSYP51 on the PVC/vacuole and forms a complex with AtSYP121 at the PM (Ebine et al., $2008,2011)$. Roles in vacuolar trafficking was shown that in the atsyp22/atvamp727 double mutant, storage vacuolar cargo such as $2 \mathrm{~s}$ albumin was secreted (Ebine et al., 2008). The double mutant contained multiple small vacuoles instead of large vacuoles found in wild-type and in atvamp727 and atsyp22 single mutants (Ebine et al., 2008), supporting that AtSYP22 and AtVAMP727 may form a SNARE complex for membrane fusion between PVC and vacuole. In addition, Rab-GTPase, Ara6 has been identified to regulate the formation of AtVamp727 SNARE complex with AtSYP121 at PM under salt stress (Ebine et al., 2011), suggesting AtVAMP727 has another role in PM, presumably triggered by salt stress. These 
results are important milestones in our understanding of vacuole biogenesis and stress response. Analyses of the diverse SNARE complexes at the tonoplast will provide further valuable information about vacuole biogenesis and vacuolar trafficking pathways possibly in relation to cell type specificity, along with plant development and growth, and plant may have developed specific traffic route to overcome environmental stress.

\section{ROLE OF PLASMA MEMBRANE SNARES}

A total of 23 SNAREs (nine Qa-SNAREs: two AtSYP11, five AtSYP12, and two AtSYP13; three Qb-SNAREs: AtNPSN1; three Qb + Qc-SNAREs: AtSNAP29, AtSNAP30, and AtSNAP33; eight R-SNARE: six AtVAMP72 and two Tomosyn) have been localized at the PM (Uemura et al., 2004; Sanderfoot, 2007). A large number of Qa-SNAREs at the PM may allow the delivery of different types of vesicles to the PM in response to specific development and biotic/abiotic cues. For example, it has been shown that AtSYP121 is involved in the regulation of $\mathrm{K}^{+}$channels and resistance against barley powdery mildew (Collins et al., 2003; Sutter et al., 2006b; Honsbein et al., 2009). However, an atsyp122 mutant did not show any defects on non-host resistance, contrary to the atsyp121 mutant, although both AtSYP121 and AtSYP122 are involved in the regulation of salicylic acid and jasmonic acid (Assaad et al., 2004; Zhang et al., 2007). Growth defects were only seen in the atsyp121/atsyp122 double mutant (Assaad et al., 2004). Therefore, AtSYP121 and AtSYP122 might have overlapping functions in plant growth and development, but distinct roles in disease resistance, presumably by mediating fusion of different types of vesicles containing cargo to resist pathogens.

Two AtSYP121 SNARE complexes have been identified with AtSNAP33 and AtVAMP721 or AtVAMP722 as components in the complexes (Kwon et al., 2008). AtSNAP33 also forms a SNARE complex with AtSYP111 and individual mutants showed incomplete cytokinesis, a process that requires massive vesicle traffic at the cell plate (Lukowitz et al., 1996; Lauber et al., 1997; Heese et al., 2001). The existence of multiple AtSNAP33 complexes suggests that this protein may have multiple roles in plant development, and transport machinery of AtSNAP33 to either cell plate during cell division or PM may rely on specific traffic machinery. For example, investigation on the trafficking of AtSYP111 during cytokinesis has established that AtSYP111 is delivered to the cell plate by secretory pathways. In mitotic cells, AtSYP111 was found to localize at the TGN and cell plate, and inhibition of ER-Golgi trafficking accumulated AtSYP111 in the ER (Reichardt et al., 2007; Chow et al., 2008). These results suggest that AtSYP111 traffics through the secretory pathway, and that distribution of AtSYP111 at the cell plate depends on a clathrin and dynamin-related protein A dependent endocytosis (Boutte et al., 2010). However, there is no evidence that AtSYP111 is directly delivered from TGN to cell plate. Considering endocytosis dependent polar distribution of auxin transporter (PIN1; Dhonukshe et al., 2008), AtSYP111 may be secreted to PM first by the default pathway and then relocalized to the cell plate by endocytosis.

Recent discovery for a role of Qa-SNAREs (AtSYP11, AtSYP12, and AtSYP13 families) at the PM supports a diversified but redundant function of Qa-SNAREs at this compartment. It was found that AtSYP132 could complement an atsyp111 mutant
(Reichardt et al., 2011). Also, replacement of the SNARE domain of AtSYP111 with that of AtSYP132 rescued the atsyp111 mutant. However, neither AtSYP121 nor the AtSYP111 chimera containing the SNARE domain of AtSYP121 was able to complement the atsyp111 mutant (Reichardt et al., 2011). These results support that while AtSYP132 may have an overlapping function with other SNAREs at the PM, AtSYP111, and AtSYP121 may have distinct roles, perhaps by forming highly specialized complexes. For example, although AtSYP111 and AtSYP121 interact with SNAP33 (Heese et al., 2001; Kwon et al., 2008), AtSYP111 may form a SNARE complex with different R-SNAREs. Identification of the R-SNAREs in the AtSYP111 and AtSYP121 complexes along with structural analyses of the SNARE complexes by X-ray crystallography may provide fundamental information on the difference between AtSYP111 and AtSYP121 SNARE complexes.

\section{CONCLUSION}

The large number of SNAREs in Arabidopsis implicates diversified roles of SNARE complexes in membrane traffic events during growth and development as well as responses to biotic and abiotic stresses. It is also important to consider that other proteins may contribute to the function of SNAREs, thus increasing the level of complexity as well as fidelity and regulation of the biological processes mediated by SNAREs. For example, the Sec1/Munc18 (SM) protein family was reported to regulate a SNARE complex formation by controlling a Qa-SNARE in mammalian and yeast cells (Malsam et al., 2008). Similarly in plants, AtSYP41 and AtSYP42 form distinct SNARE complexes at different subdomains of TGN with AtSYP61, AtVTI12, and a SM protein, AtVPS45 (Bassham et al., 2000; Sanderfoot et al., 2001a). AtVPS45 RNAi plants showed decreased amount of AtSYP41 compared to wildtype, suggesting that AtVPS45 may be important for the stability of AtSYP41 (Zouhar et al., 2009). These findings support that SM proteins regulate SNARE-mediated membrane fusion, as well as stability or turnover of SNAREs. In Arabidopsis, six putative Sec1like proteins in the SM protein family have been predicted to exist (Schwacke et al., 2003). Thus, investigation of the uncharacterized SM proteins will be necessary to define one of the possible regulatory mechanisms of SNARE complexes. Related to this, tethering factors and GTPases can play important roles in membrane fusion (Malsam et al., 2008). In yeast, tethering factors are involved in the SNARE complex assembly and docking for membrane fusion by interacting with GTPases (Price et al., 2000; Seals et al., 2000; Shorter et al., 2002), supporting that SNARE complex assembly is a multifaceted process based on the interplay of multiple components. Therefore, a study focused on SNARE specificity in vesicle trafficking should take into account an analysis of several components for membrane fusion to better define an overall picture of the function of the SNAREs.

So far, valuable approaches to characterize the role of SNAREs have been developed and have provided important data in plants. However, there are still several unsolved questions on the function of SNAREs and the nature of the complexes that they can form in vivo in plant cells. Arabidopsis has a large number of SNAREs and several SNARE complexes that partially share the same components. We need to understand the physiological relevance of the number of SNARE and of the SNARE complexes. 
With no doubt, we are living in an exciting moment for research in plants thanks to a wealth of genetics, genomics, and molecular resources. In vitro fusion assays using SNAREs and other regulatory proteins may help specify the roles of SNARE complexes with similar but not identical components. The combination of in vitro and in vivo studies on cargo trafficking and membrane fusion will also provide important insights into the mechanisms

\section{REFERENCES}

Ahmed, S. U., Barpeled, M., and Raikhel, N. V. (1997). Cloning and subcellular location of an Arabidopsis receptor-like protein that shares common features with proteinsorting receptors of eukaryotic cells. Plant Physiol. 115, 311-312.

Assaad, F. F., Qiu, J. L., Youngs, H., Ehrhardt, D., Zimmerli, L., Kalde, M., Wanner, G., Peck, S. C., Edwards, H., Ramonell, K., Somerville, C. R., and Thordal-Christensen, H. (2004). The PEN1 syntaxin defines a novel cellular compartment upon fungal attack and is required for the timely assembly of papillae. Mol. Biol. Cell 15, 5118-5129.

Bassham, D. C., Sanderfoot, A. A., Kovaleva, V., Zheng, H. Y., and Raikhel, N. V. (2000). AtVPS45 complex formation at the transGolgi network. Mol. Biol. Cell 11, 2251-2265.

Blatt, M. R. (2000). Cellular signaling and volume control in stomatal movements in plants. Annu. Rev. Cell Dev. Biol. 16, 221-241.

Bock, J. B., Matern, H. T., Peden, A. A., and Scheller, R. H. (2001). A genomic perspective on membrane compartment organization. Nature 409, 839-841.

Boutte, Y., Frescatada-Rosa, M., Men, S. Z., Chow, C. M., Ebine, K., Gustavsson, A., Johansson, L., Ueda, T., Moore, I., Jurgens, G., and Grebe, M. (2010). Endocytosis restricts Arabidopsis KNOLLE syntaxin to the cell division plane during late cytokinesis. EMBO J. 29, 546-558.

Bubeck, J., Scheuring, D., Hummel, E., Langhans, M., Viotti, C., Foresti, O., Denecke, J., Banfield, D. K., and Robinson, D. G. (2008). The syntaxins SYP31 and SYP81 control ER-Golgi trafficking in the plant secretory pathway. Traffic 9, 1629-1652.

Chatre, L., Brandizzi, F., Hocquellet, A., Hawes, C., and Moreau, P. (2005). Sec22 and Memb11 are v-SNAREs of the anterograde endoplasmic reticulum-Golgi pathway in tobacco leaf epidermal cells. Plant Physiol. 139, 1244-1254.

Chow, C. M., Neto, H., Foucart, C., and Moore, I. (2008). Rab-A2 and
Rab-A3 GTPases define a transGolgi endosomal membrane domain in Arabidopsis that contributes substantially to the cell plate. Plant Cell 20, 101-123.

Collins, N. C., Thordal-Christensen, H., Lipka, V., Bau, S., Kombrink, E., Qiu, J. L., Huckelhoven, R., Stein, M., Freialdenhoven, A., Somerville, S. C., and Schulze-Lefert, P. (2003). SNARE-protein-mediated disease resistance at the plant cell wall. Nature 425, 973-977.

Dettmer, J., Hong-Hermesdorf, A., Stierhof, Y. D., and Schumacher, K. (2006). Vacuolar $\mathrm{H}^{+}$-ATPase activity is required for endocytic and secretory trafficking in Arabidopsis. Plant Cell 18, 715-730.

Dhonukshe, P., Tanaka, H., Goh, T., Ebine, K., Mahonen, A. P., Prasad, K., Blilou, I., Geldner, N., Xu, J., Uemura, T., Chory, J., Ueda, T., Nakano, A., Scheres, B., and Friml, J. (2008). Generation of cell polarity in plants links endocytosis, auxin distribution and cell fate decisions. Nature 456, U962-U975.

Drakakaki, G., Van De Ven, W., Pan, S., Miao, Y., Wang, J., Keinath, N. K., Weatherly, B., Jiang, L., Schumacher, K., Hicks, G., and Raikhel, N. (2011). Isolation and proteomic analysis of the SYP61 compartment reveal its role in exocytic trafficking in Arabidopsis. Cell Res. 1-12.

Ebine, K., Fujimoto, M., Okatani, Y., Nishiyama, T., Goh, T., Ito, E., Dainobu, T., Nishitani, A., Uemura, T., Sato, M. H., Thordal-Christensen, H., Tsutsumi, N., Nakano, A., and Ueda, T. (2011). A membrane trafficking pathway regulated by the plant-specific RAB GTPase ARA6. Nat. Cell Biol. 13, U853-U859.

Ebine, K., Okatani, Y., Uemura, T., Goh, T., Shoda, K., Niihama, M., Morita, M. T., Spitzer, C., Otegui, M. S., Nakano, A., and Ueda, T. (2008). A SNARE complex unique to seed plants is required for protein storage vacuole biogenesis and seed development of Arabidopsis thaliana. Plant Cell 20, 3006-3021.

El-Kasmi, F., Pacher, T., Strompen, G., Stierhof, Y. D., Müller, L. M., Koncz, C., Mayer, U., and Jürgens, G. (2011). Arabidopsis SNARE protein

and regulatory pathways of vesicle transport along with a better understanding of their role in plant growth, development, and response to stress.

\section{ACKNOWLEDGMENT}

This work is supported by the Great Lake Bioenergy Research Center (GLBRC) under the grant (DE-FC02-07ER64494).

SEC22 is essential for gametophyte development and maintenance of Golgi-stack integrity. Plant J. 66, 268-279.

Fasshauer, D., Sutton, R. B., Brunger, A. T., and Jahn, R. (1998). Conserved structural features of the synaptic fusion complex: SNARE proteins reclassified as Q- and R-SNAREs. Proc. Natl. Acad. Sci. U.S.A. 95, 15781-15786.

Foresti, O., Dasilva, L. L., and Denecke, J. (2006). Overexpression of the Arabidopsis syntaxin PEP12/SYP21 inhibits transport from the prevacuolar compartment to the lytic vacuole in vivo. Plant Cell 18, 2275-2293.

Hara-Nishimura, I., Shimada, T., Hatano, K., Takeuchi, Y., and Nishimura, M. (1998). Transport of storage proteins to protein storage vacuoles is mediated by large precursor-accumulating vesicles. Plant Cell 10, 825-836.

Hayashi, Y., Yamada, K., Shimada, T., Matsushima, R., Nishizawa, N. K., Nishimura, M., and HaraNishimura, I. (2001). A proteinasestoring body that prepares for cell death or stresses in the epidermal cells of Arabidopsis. Plant Cell Physiol. 42, 894-899.

Heese, M., Gansel, X., Sticher, L., Wick, P., Grebe, M., Granier, F., and Jurgens, G. (2001). Functional characterization of the KNOLLEinteracting t-SNARE AtSNAP33 and its role in plant cytokinesis. J. Cell Biol. 155, 239-249.

Honsbein, A., Sokolovski, S., Grefen, C., Campanoni, P., Pratelli, R., Paneque, M., Chen, Z. H., Johansson, I., and Blatt, M. R. (2009). A tripartite SNARE- $\mathrm{K}^{+}$channel complex mediates in channel-dependent $\mathrm{K}^{+}$nutrition in Arabidopsis. Plant Cell 21, 2859-2877.

Kwon, C., Neu, C., Pajonk, S., Yun, H. S., Lipka, U., Humphry, M., Bau, S., Straus, M., Kwaaitaal, M., Rampelt, H., El Kasmi, F., Jurgens, G., Parker, J., Panstruga, R., Lipka, V., and Schulze-Lefert, P. (2008). Cooption of a default secretory pathway for plant immune responses. Nature 451, 835-840.

Lauber, M. H., Waizenegger, I., Steinmann, T., Schwarz, H., Mayer, U.,
Hwang, I., Lukowitz, W., and Jurgens, G. (1997). The Arabidopsis KNOLLE protein is a cytokinesisspecific syntaxin. J. Cell Biol. 139, 1485-1493.

Leshem, Y., Golani, Y., Kaye, Y., and Levine, A. (2010). Reduced expression of the $\mathrm{v}$-SNAREs AtVAMP71/AtVAMP7C gene family in Arabidopsis reduces drought tolerance by suppression of abscisic acid-dependent stomatal closure. $J$. Exp. Bot. 61, 2615-2622.

Lukowitz, W., Mayer, U., and Jurgens, G. (1996). Cytokinesis in the Arabidopsis embryo involves the syntaxinrelated KNOLLE gene product. Cell 84, 61-71.

Malsam, J., Kreye, S., and Sollner, T. H. (2008). Membrane fusion: SNAREs and regulation. Cell. Mol. Life Sci. 65 , 2814-2832.

Mancias, J., and Goldberg, J. (2008). Structural basis of cargo membrane protein discrimination by the human COPII coat machinery. EMBO J. 27, 2918-2928.

McNew, J. A., Parlati, F., Fukuda, R., Johnston, R. J., Paz, K., Paumet F., Sollner, T. H., and Rothman J. E. (2000). Compartmental specificity of cellular membrane fusion encoded in SNARE proteins. Nature 407, 153-159.

Nakamura, K., Matsuoka, K., Mukumoto, F., and Watanabe, N. (1993). Processing and transport to the vacuole of a precursor to sweetpotato sporamin in transformed tobacco cell line-by-2. J. Exp. Bot. 44, 331-338.

Price, A., Wickner, W., and Ungermann, C. (2000). Proteins needed for vesicle budding from the Golgi complex are also required for the docking step of homotypic vacuole fusion. J. Cell Biol. 148, 1223-1229.

Reichardt, I., Slane, D., El Kasmi, F., Knöll, C., Fuchs, R., Mayer, U., Lipka, V., and Jürgens, G. (2011) Mechanisms of functional specificity among plasma-membrane syntaxins in Arabidopsis. Traffic 12, 1269-1280.

Reichardt, L., Stierhof, Y. D., Mayer, U., Richter, S., Schwarz, H., Schumacher, K., and Jurgens, G. (2007). Plant cytokinesis requires de novo secretory trafficking but not endocytosis. Curr. Biol. 17, 2047-2053. 
Robinson, D. G., Herranz, M. C., Bubeck, J., Pepperkok, R., and Ritzenthaler, C. (2007). Membrane dynamics in the early secretory pathway. CRC Crit. Rev. Plant Sci. 26, 199-225.

Saito, C., and Ueda, T. (2009). Functions of Rab and SNARE proteins in plant life. Int. Rev. Cell Mol. Biol. 274, 183-233.

Sanderfoot, A. (2007). Increases in the number of SNARE genes parallels the rise of multicellularity among the green plants. Plant Physiol. 144, 6-17.

Sanderfoot, A. A., Kovaleva, V., Bassham, D. C., and Raikhel, N. V. (2001a). Interactions between syntaxins identify at least five SNARE complexes within the Golgi/prevacuolar system of the Arabidopsis cell. Mol. Biol. Cell 12, 3733-3743.

Sanderfoot, A. A., Pilgrim, M., Adam, L., and Raikhel, N. V. (2001b). Disruption of individual members of Arabidopsis syntaxin gene families indicates each has essential functions. Plant Cell 13, 659-666.

Scheuring, D., Viotti, C., Kruger, F., Kunzl, F., Sturm, S., Bubeck, J., Hillmer, S., Frigerio, L., Robinson, D. G., Pimpl, P., and Schumacher, K. (2011). Multivesicular bodies mature from the trans-Golgi network/early endosome in Arabidopsis. Plant Cell 23, 3463-3481.

Schwacke, R., Schneider, A., Van Der Graaff, E., Fischer, K., Catoni, E., Desimone, M., Frommer, W. B., Flugge, U. I., and Kunze, R. (2003). ARAMEMNON, a novel database for Arabidopsis integral membrane proteins. Plant Physiol. 131, $16-26$

Seals, D. F., Eitzen, G., Margolis, N., Wickner, W. T., and Price, A. (2000). AYpt/Rab effector complex containing the Secl homolog Vps33p is required for homotypic vacuole fusion. Proc. Natl. Acad. Sci. U.S.A. 97, 9402-9407.

Shirakawa, M., Ueda, H., Shimada, T., Koumoto, Y., Shimada, T. L., Kondo, M., Takahashi, T., Okuyama, Y., Nishimura, M. and Hara-Nishimura, I. (2010). Arabidopsis Qa-SNARE SYP2 proteins localized to different subcellular regions function redundantly in vacuolar protein sorting and plant development. Plant J. 64, 924-935.

Shorter, J., Beard, M. B., Seemann, J., Dirac-Svejstrup, A. B., and Warren, G. (2002). Sequential tethering of Golgins and catalysis of SNAREpin assembly by the vesicle-tethering protein p115. J. Cell Biol. 157, 45-62.

Sirichandra, C., Wasilewska, A., Vlad, F., Valon, C., and Leung, J. (2009). The guard cell as a single-cell model towards understanding drought tolerance and abscisic acid action. J. Exp. Bot. 60, 1439-1463.

Sollner, T., Whitehart, S., Brunner, M., Erdjumentbromage, H., Geromanos, S., Tempst, P., and Rothman, J. (1993). SNAP receptors implicated in vesicle targeting and fusion. Nature 362, 318-324.

Spang, A., and Schekman, R. (1998). Reconstitution of retrograde transport from the Golgi to the ER in vitro. J. Cell Biol. 143, 589-599.

Sutter, J. U., Campanoni, P., Blatt, M. R., and Paneque, M. (2006a). Setting SNAREs in a different wood. Traffic 7, 627-638.

Sutter, J. U., Campanoni, P., Tyrrell, M., and Blatt, M. R. (2006b). Selective mobility and sensitivity to SNAREs is exhibited by the Arabidopsis $\mathrm{KAT} 1 \mathrm{~K}^{+}$channel at the plasma membrane. Plant Cell 18, 935-954.
Suwastika, I. N., Uemura, T., Shiina, T. H Sato, M., and Takeyasu, K. (2008). SYP71, a plant-specific Qc-SNARE protein, reveals dual localization to the plasma membrane and the endoplasmic reticulum in Arabidopsis. Cell Struct. Funct. 33, 185-192.

Toyooka, K., Goto, Y., Asatsuma, S., and Matsuoka, K. (2007). SCAMP-containing vesicle cluster (SVC), a post-Golgi exocytotic organelle. Plant Cell Physiol. 48, S122.

Toyooka, K., Okamoto, T., and Minamikawa, T. (2000). Mass transport of proform of a KDEL-tailed cysteine proteinase (SH-EP) to protein storage vacuoles by endoplasmic reticulum-derived vesicle is involved in protein mobilization in germinating seeds. J. Cell Biol. 148, 453-464.

Tsui, M. M., Tai, W. C., and Banfield, D. K. (2001). Selective formation of Sed5p-containing SNARE complexes is mediated by combinatorial binding interactions. Mol. Biol. Cell 12, 521-538.

Uemura, T., Morita, M. T., Ebine, K., Okatani, Y., Yano, D., Saito, C., Ueda, T., and Nakano, A. (2010). Vacuolar/pre-vacuolar compartment Qa-SNAREs VAM3/SYP22 and PEP12/SYP21 have interchangeable functions in Arabidopsis. Plant J. 64 864-873.

Uemura, T., Ueda, T., Ohniwa, R. L., Nakano, A., Takeyasu, K., and Sato, M. H. (2004). Systematic analysis of SNARE molecules in Arabidopsis dissection of the post-Golgi network in plant cells. Cell Struct. Funct. 29, 49-65.

Yano, D., Sato, M., Saito, C., Sato, M. H., Morita, M. T., and Tasaka, M. (2003). A SNARE complex containing SGR3/AtVAM3 and ZIG/VTI11 in gravity-sensing cells is important for Arabidopsis shoot gravitropism.
Proc. Natl. Acad. Sci. U.S.A. 100, 8589-8594.

Zhang, Z. G., Feechan, A., Pedersen, C., Newman, M. A., Qiu, J. L., Olesen, K. L., and Thordal-Christensen, H. (2007). A SNARE-protein has opposing functions in penetration resistance and defence signalling pathways. Plant J. 49, 302-312.

Zhu, J. H., Gong, Z. Z., Zhang, C. Q., Song, C. P., Damsz, B., Inan, G. Koiwa, H., Zhu, J. K., Hasegawa, P. M., and Bressan, R. A. (2002). OSM1/SYP61: a syntaxin protein in Arabidopsis controls abscisic acidmediated and non-abscisic acidmediated responses to abiotic stress. Plant Cell 14, 3009-3028.

Zouhar, J., Rojo, E., and Bassham, D. C. (2009). AtVPS45 Is a positive regulator of the SYP41/SYP61/VTI12 SNARE complex involved in trafficking of vacuolar cargo. Plant Physiol. 149, 1668-1678.

Conflict of Interest Statement: The authors declare that the research was conducted in the absence of any commercial or financial relationships that could be construed as a potential conflict of interest.

Received: 04 November 2011; accepted: 25 January 2012; published online: 10 February 2012.

Citation: Kim S-J and Brandizzi F (2012) News and views into the SNARE complexity in Arabidopsis. Front. Plant Sci. 3:28. doi: 10.3389/fpls.2012.00028

This article was submitted to Frontiers in Plant Cell Biology, a specialty of Frontiers in Plant Science.

Copyright (c) 2012 Kim and Brandizzi. This is an open-access article distributed under the terms of the Creative Commons Attribution Non Commercial License, which permits non-commercial use, distribution, and reproduction in other forums, provided the original authors and source are credited. 